BNL-47065

Informal Report

\title{
Polarized Protons and RHIC
}

Based on a presentation to the Brookhaven PAC in August 1991

G. Bunce for the RHIC Spin Collaboration

December 1991 
This report was prepared as an account of work sponsored by an agency of the United States Government. Neither the Ur ted States Government nor any agency thereof, nor any of their employees, nor any of their contractors, subcontractors, or their employees makes any warranty, express or implied, or assumes any legal liability or responsibility for the accuracy, completeness, or usefulness of any information, apparatus, product or process disclosed, or represents that its use would not infringe privately owned rights. Reference herein to any specific commercial product, process, or service by trade name, trademark, manufacturer, or otherwise, does not necessarily constitute or imply its endorsement, recommendation, or favoring by the United states Government or any azency thereof. The views and opinions of authors expressed herein do not necessarily state or reflect those of the United States Government or any agency, contractor, or subcontractor thereof. 
BNL-47065

EP\&S 91-4

Informal Report

\section{Polarized Protons and RHIC}

Based on a presentation to the Brookhaven PAC in August 1991

G. Bunce for the RHIC Spin Collaboration

December 1991

AGS Department

Brookhaven National Laboratory

Associated Universities, Inc.

Upton, Long Island, New York 11973

Under Contract No. DE-AC02-76CH00016 with the UNITED STATES DEPARTMENT OF ENERGY

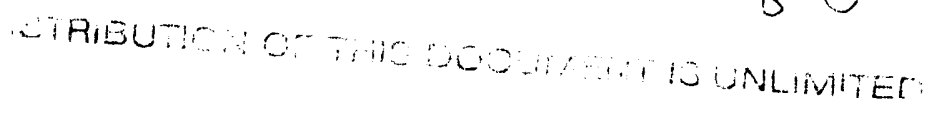




\section{Polarized Protons and RHIC}

Based on a preserfation to the Brookhaven PAC in August 1991

G. Bunce for the RHIC Spin Collaboration't

\section{Introduction}

RHIC, the heavy ion collider being built at Brookhaven, offers an exciting opportunity to collide highly polarized protons at high energy and luminosity. This new facility would combine the existing AGS polarized proton capability with the new Booster/Accumulator and spin rotators (Siberian Snakes) to achieve collisions between intense breams of polarized protons at a collision energy of $500 \mathrm{GeV}$. At this energy and the expected lunninosity of $2 \times 10^{32} \mathrm{~cm}^{2} /$ second physics probes will include high $\mathrm{p}_{\mathrm{T}}$ jets, direct photons, Drell-Yanl, $\mathrm{W}^{ \pm}$, and heavy quarks. The accessible physics includes study of the spin content of the proton, particularly gluon and antiquark polarization, study of large PQCD-predicted asymmetries for parton-parton subprocesses, and parity violation studies and searches. The proton spin direction at a RHIC crossing can be longitudinal or transverse and can alternate bunch-to-bunch giving exquisite control of systematic errors. At RHIC double spin experiments can be done with pure beams and the energy and luminosity open a new domain for probing the physics of spin.

An international collaboration is forming which proposes to exploit the unique physics available from a polarized RHIC. Important steps, leading to a polarized RHIC, have been taken. The AGS has already accelerated polarized protons. A new Booster/Accumulator has been commissioned. A beautiful series of machine experiments at Indiana University have verified that spin rotators (Siberian Snakes) indeed remove spin resonance behavior, which is the key to achieving polarized proton acceleration to high energy. E880, an accelerator experiment which will build, install, and test a Siberian Snake in the AGS, was approved by the Brookhaven PAC in August 1991. The snake will be installed in the AGS in the summer of 1993. RHIC construction has started, with heavy ion experiments to begin in 1997.

The RHIC Spin Collaboration (RSC) presented a letter of intent to the PAC, also in August this year, and was encouraged to submit a proposal. RSC intends to submit the proposal 
for the spin program in the fall of 1992 and has the goal of colliding polarized protons early in RHIC's running schedule.

\section{Technical Advances in Spin}

The experiments at IUCF at Indiana University ${ }^{2}$ have shown that spin rotators ${ }^{3}$ cancel spin resonances, as predicted. An example is shown in Fig. 1, where a spin resonance which exists near a vertical tune of $v_{y}=5.13$ is canceled by a snake. It was also shown that a partial spin rotation cancels weaker spin resonances--this technique will be used in the AGS. ${ }^{4}$

The proposed polarized RHIC complex is shown in Fig. 2. As indicated there, $2 \times 10^{11}$ polarized protons per bunch will give a luminosity of $2 \times 10^{32} \mathrm{~cm}^{2} /$ second for collisions at $\sqrt{\mathrm{s}}=$ $500 \mathrm{GeV}$.

\section{Polarized Protons and Polarized Partons}

Deep inelastic scattering experiments with beams of electrons (SLAC) and muons (CERN) have shown that the valence quarks in the polarized proton are quite polarized for quarks which carry a substantial fraction of the proton momentum. ${ }^{5}$ This is shown in Fig. 3, where $A_{1}$ is the quark polarization and $\mathrm{x}$ is the quark momentum fraction.

The average contribution of the quark spin to the proton spin is ciominated by the quarks with low momenta. A surprising result from CERN was that this average quark spin is small, leading to the possibility of large gluon and/or antiquark polarization.

RSC will be able to select scattering at large quark momenta to study scattering of highly polarized quarks, and will study the gluon and antiquark polarization in the proton. If either turns out to be polarized, and if the CERN experiment is correct one must be polarized, RSC will be able to scatter beams of polarized gluons or antiquarks as well as polarized quarks. 


\section{Spin Physics at High_ $\mathbb{R}_{1}$}

The RHIC Spin Collaboration began with an important workshop at Penn State University in November 1990. This was attended by about 80 physicists and the proceedings ${ }^{6}$ describes a $^{2}$ wide range of exciting physics. In addition, J. Soffer et al. have published a study of physics that would be accessible at a polarized RHIC. ${ }^{7}$ Here we will discuss only a few of the physics topics.

It is clear that the first emphasis will be to deternine the polarization of the quarks, gluons, and antiquarks in the polarized protons. An asymmetry at the particle level derives from parton polarizations folded with asymmetries in the parton-parton scattering. For direct photon production, which is expected to be dominated by quark-gluon scattering (Fig. 4) and is therefore a clean probe of gluon polarization, the required quark asymmetry is $g_{1}$, the parameter already measured in deep inelastic scattering. The PQCD prediction for the quark-gluon to photon subprocess is very large (curve $\mathrm{E}$ in Fig. 5), so this process is very sensitive to the gluon asymmetry. Figure 4 shows the expected asymmetry for direct photons under two models--one with a large gluon asymmetry which would account for the missing average quark contribution to the proton spin, and the other with a small standard model for gluon asymmetry. Superimposed is an error bar representing the sensitivity of a one month run at RHIC, assuming a detector with full azimuthal coverage and $45^{\circ}$ coverage in scattering angle $(\Delta \eta=1) . \Delta A_{L L}=$ .02 at $\mathrm{p}_{\mathrm{T}}=20 \mathrm{GeV}$ is expected.

Jet production asymmetry is also very sensitive to the gluon asymmetry. For $p_{\mathrm{T}}$ below about $50 \mathrm{GeV}$, jets are expected to come from gluon-gluon scattering. The asymmetry for gluongluon scattering from $\mathrm{PQCD}$ calculations is very large--curve $\mathrm{A}$ in Fig. 5. The jet asymmetry is shown in Fig. 6 for the two gluon models mentioned above. The sensitivity for a one month run at RHIC is $\Delta \mathrm{A}_{\mathrm{LL}}=.005$ at $\mathrm{P}_{\mathrm{T}}=50$, as shown in the figure.

The PQCD predictions in Fig. 5 can be tested directly with beams of polarized quarks, gluons and antiquarks. Drell-Yan pairs are sensitive probes of antiquark polarization. Parity violation can be measured for $\mathrm{W}$ decays; it can be used to statistically isolate $\mathrm{W}$ decays which 
otherwise have serious background, for example $W \rightarrow 2$ jets and $W \rightarrow$ lower energy electrons; parity violation can be used to search for new physics signals--a compositeness search this way might complement higher energy collider searches which look for a flattening of jet production at high $\mathrm{p}_{\mathrm{T}}$.

Experiments to study transverse polarization effects are also very attractive. ${ }^{8}$ Consideration of the proton spin puzzle has led to the realization that the proton spin structure can be quite rich. For example, RSC can measure two transverse spin effects individually which had been assumed to be equal: the difference in transversity of valence quarks and the transverse quark spin distribution including sea quarks. ${ }^{9}$

\section{$\underline{\text { Detectors }}$}

There is considerable work to do to define the spin detector (or detectors), and to verify sensitivities with simulation programs. It is clear that the probes to be emphasized are jets, direct photons, W, and Drell-Yan pairs. It is surprising that the RHIC detectors (that is, for heavy ion

physics) may be a reasonable match for doing high luminosity proton physics. A large detector granularity is necessary for RHIC with low luminosity, and this "matches" the luminosity $x$ multiplicity of high luminosity proton-proton collisions. Thus, RSC is considering using, possibly augmenting, RHIC detectors for spin physics. Also, an ideal jet detector might be a version of UA2 at CERN. This approach is also being considered.

\section{Plans}

E880, the experiment which will install a partial snake in the AGS, is proceeding. The snake installation will be in the summer of 1993. RSC is studying detector approaches and expects to decide on its direction by January 1992. We anticipate submitting a proposal in the fall of 1992. When the proposal is approved we will seek funding. It is important that the possibilities with a polarized RHIC be brought to the physics community, and it is important to continue to develop the collaboration. 


\section{P.eferences}

1. Present members of the RHIC Spin Collaboration:

Argonne M. Beddo, D. Hill, D. Grosnick, D. Lopiano, H. Spinka, D. Underwood, A. Yokosawa.

BNL G. Bunce, A. Carroll, E. Courant, R. Fernow, Y.Y. Lee, D. Lowenstein, Y. Makdisi, L. Ratner, T. Roser, A. Sambamurti, M. Tannenbaum

Dubna $\quad$ A.V. Efremov

Indiana S.Y. Lee

Helsinki N. Törnquist

ITEP Y. Arestov, B.V. Chuiko, A.M. Davidenko, A.A. Derevschikov, O.A. Grachov, A.K. Likhoded, A.P. Meschanin, S.B. Nurushev, V.L. Rykov, A.G. Ufintzev, A.N. Vasiliev

KEK S. Hiramatsu, Y. Mori, H. Sato

Kyoto H. Enyo, K. Imai, A. Masaike

Marseille J. Soffer

MIT R. Jaffe

Padova M. Pusterla

Penn State J. Collins, S. Heppelmann, G. Ladinsky, E. Minor, R. Robinett

Unaffiliated D. Sivers

Trieste A. Penzo, P. Schiavon

Genova M. Conte

UCLA $\quad$ G. Igo

2. A.D. Krisch et al, Phys. Rev. Lett. 63, 1137 (1989); J.E. Goodwin et al., Phys. Rev. Lett. 64, 2779 (1990). 
3. Siberian snakes: Ya. S. Derbenev and A.M. Kondratenko, Part. Accel. 8, 115 (1978).

4. The partial snake was first discussed by T. Roser in Proc. 8th High Energy Spin Physics Symposium, 1989, edited by K. Heller, AIP Conf. Proc. 187, p. 1442.

5. M.J. Alguard et al., Phys. Rev. Lett. 37, 1261 (1976); G. Baum et al., Phys. Rev. Lett. 51, 1135 (1983); EMC, J. Ashman et al., Phys. Lett. B206, 364 (1988).

6. Penn State Polarized Collider Workshop Proceedings, AIP Proceedings 223, J.C. Collins, S. Heppelmann, and R.W. Robinett editors (1991).

7. C. Bourrely, J. Ph. Guillet, and J. Soffer, Nuclear Phys. B361, 71 (1991).

8. J. Ralston and D.E. Soper, Nuclear Phys. B152, 109 (1979); J.L. Cortes, B. Pire and J.P. Ralston, p. 184 in ref. 6.

9. R.L. Jaffe and X. Ji, Phys. Rev. Lett. $\underline{67}, 552$ (1991). 


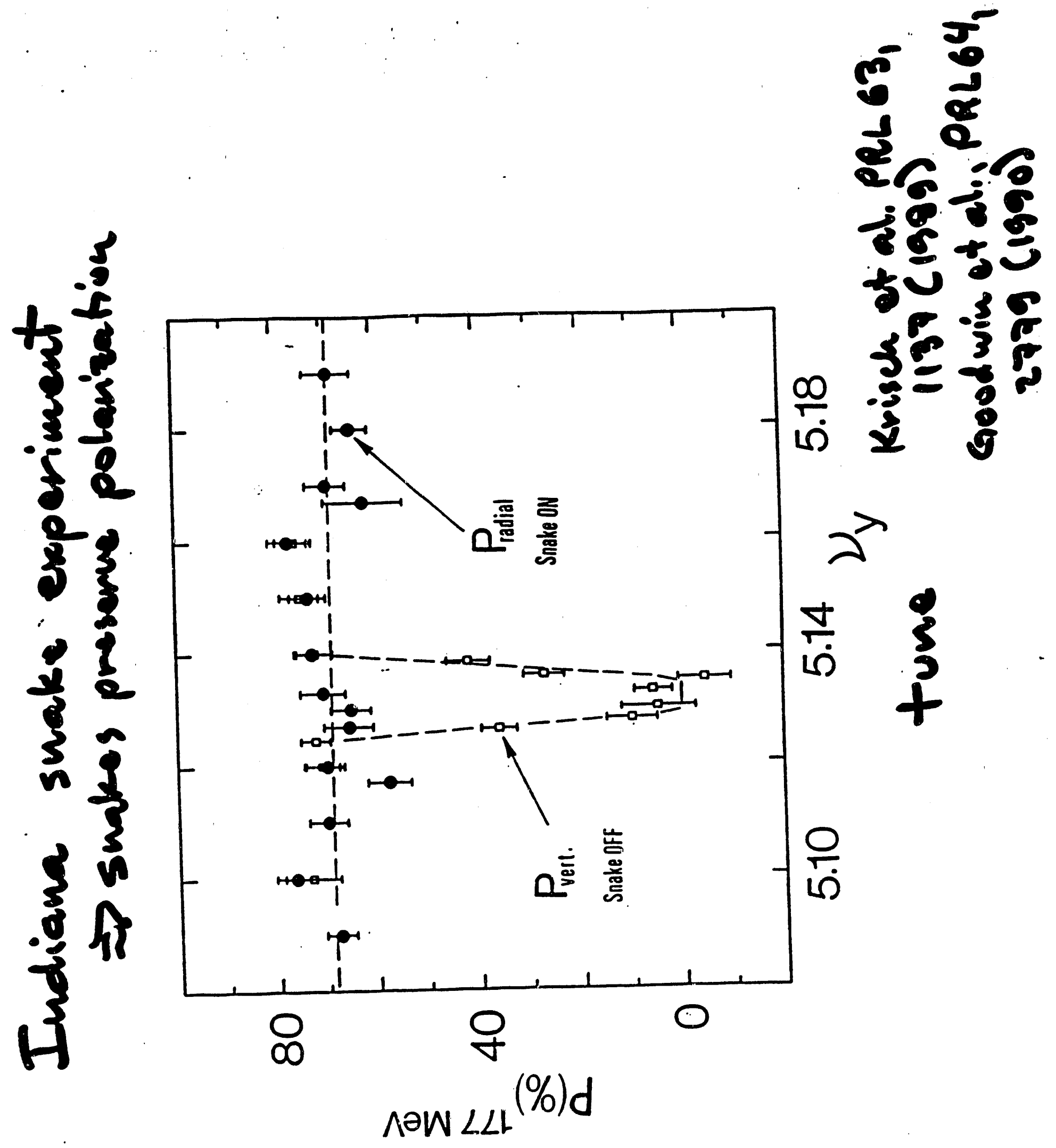

Fig. 1 


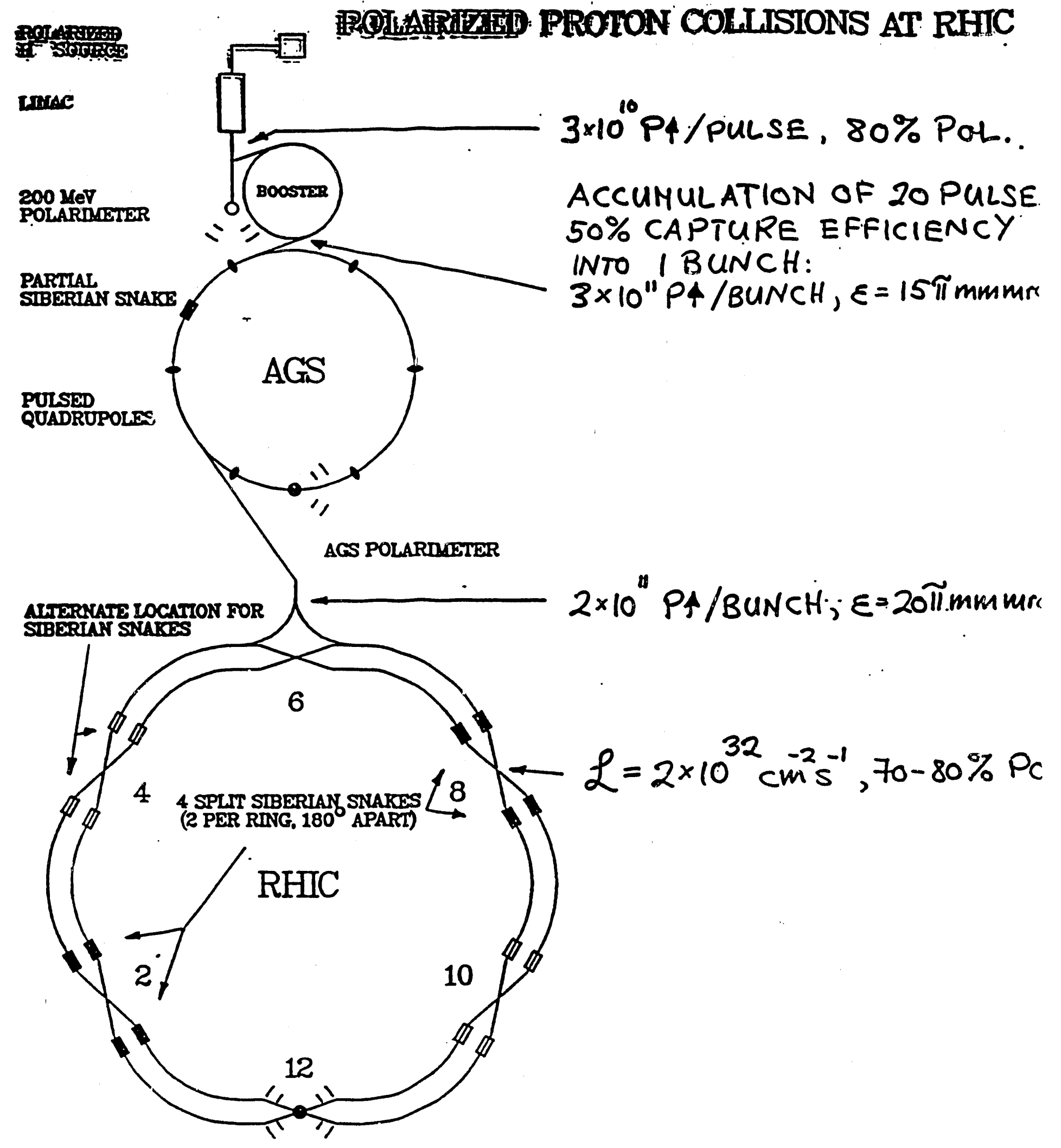

RHIC POLARIUETERR 


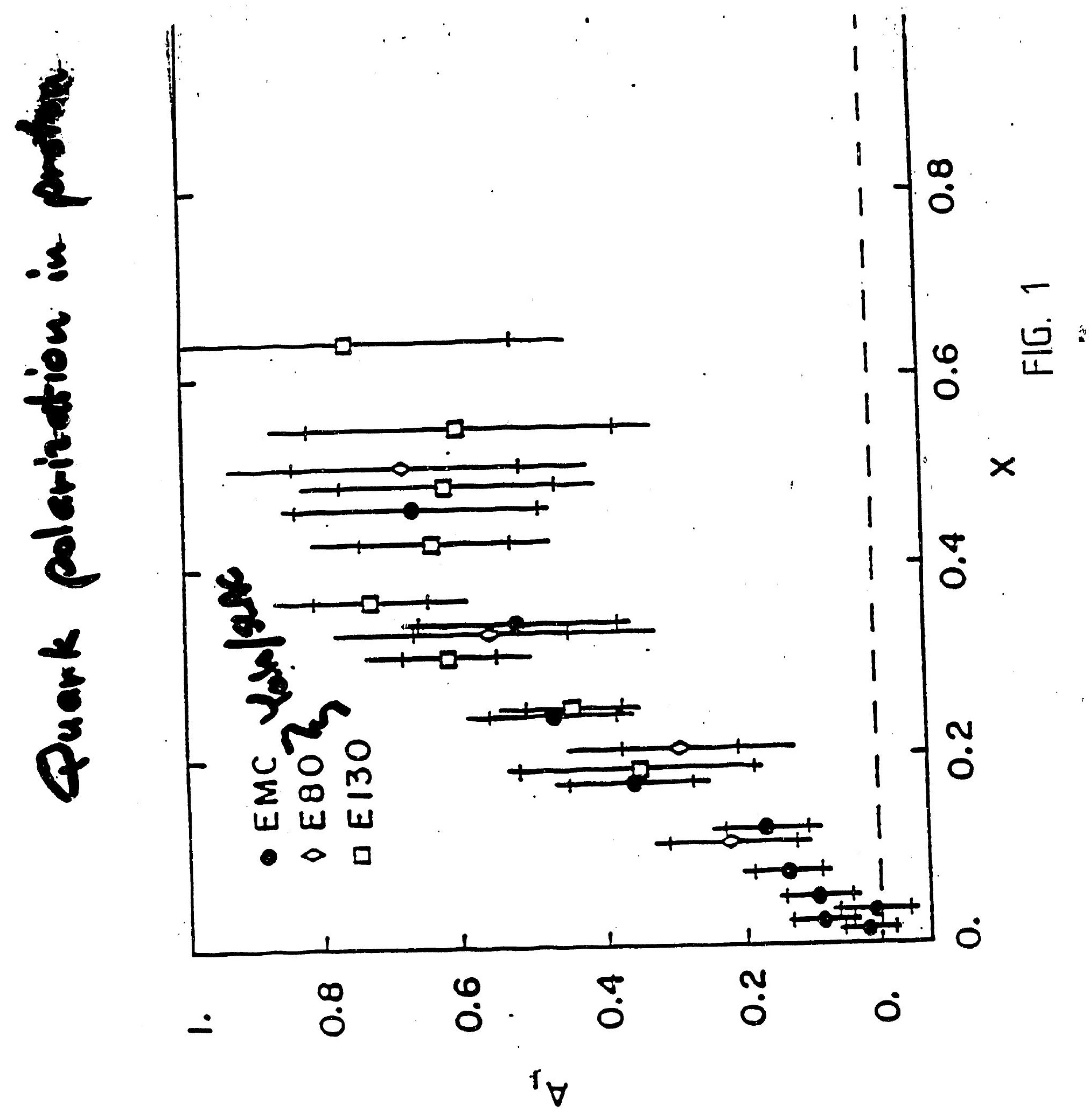

Fig. 3 
Ormest phaton semastivity to gluon polacieation

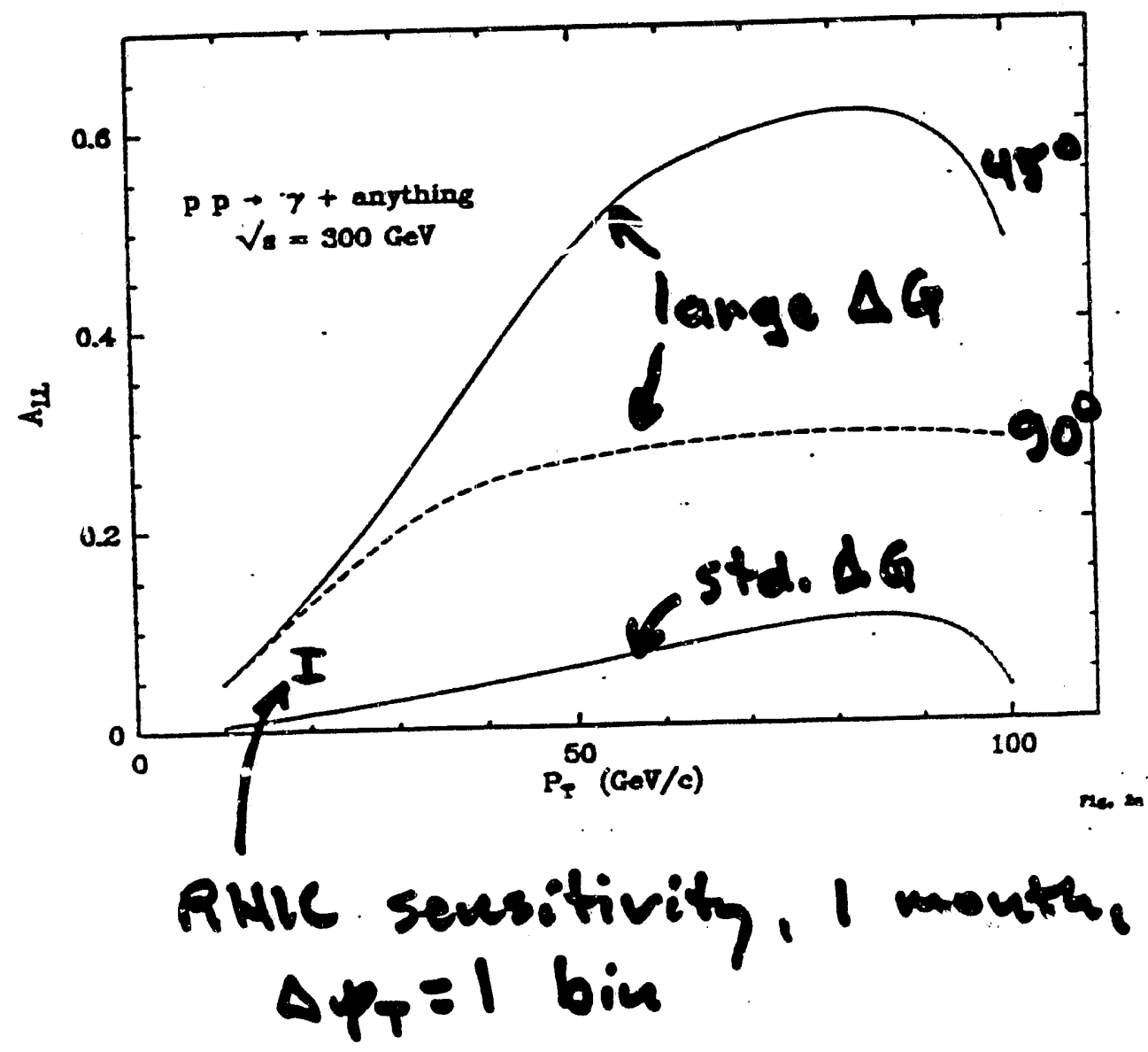

Note: $A_{u L} d \sigma \approx \int d x_{a} d x_{b}\left[g,\left(x_{a 1} Q^{2}\right) \Delta G\left(x_{b}, a^{2}\right)\right.$.

$$
\text { - } \left.a_{L b}^{28} \tilde{\sigma}^{29}\right]
$$

Fig. 4 

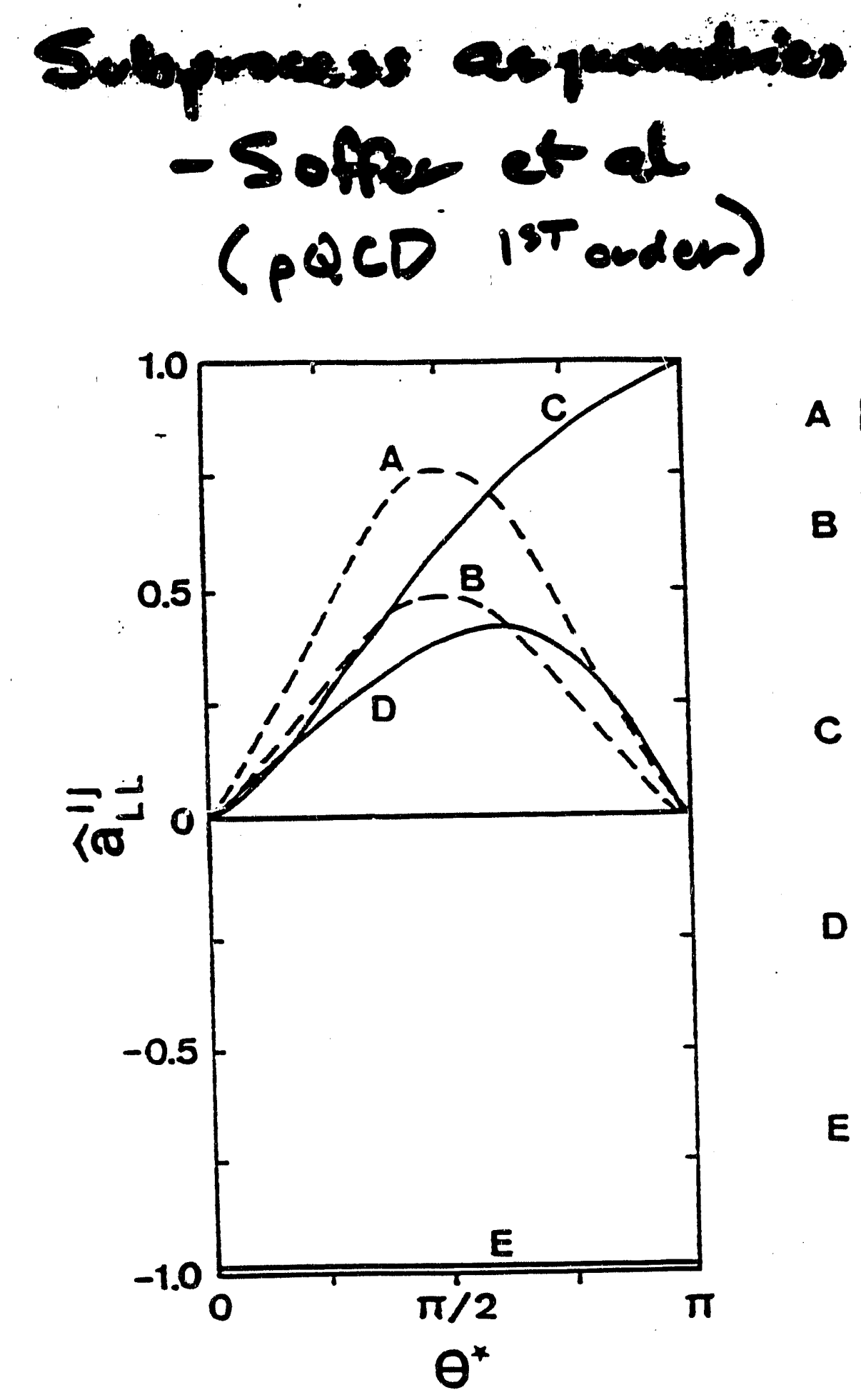

$A[9 g-9 g$

$B\left[\begin{array}{l}d d \rightarrow d d \\ u u \rightarrow u u\end{array}\right.$

$C\left[\begin{array}{l}u d \rightarrow u d \\ u \bar{d} \rightarrow u \bar{d} \\ d \bar{u} \rightarrow d \bar{u} \\ q g \rightarrow q g \\ q g \rightarrow q \gamma\end{array}\right.$

$D\left[\begin{array}{l}u \bar{u} \rightarrow u \bar{u} \\ d \bar{d} \rightarrow d \bar{d}\end{array}\right.$

$E\left[\begin{array}{l}y g \rightarrow q \bar{q} \\ q \bar{q} \rightarrow g \bar{g} \\ u \bar{u} \rightarrow d \bar{d} \\ d \bar{d}=u \bar{u} \\ q \bar{q} \rightarrow g \gamma\end{array}\right.$

Fig. 5 
common romitedion from Jet Production

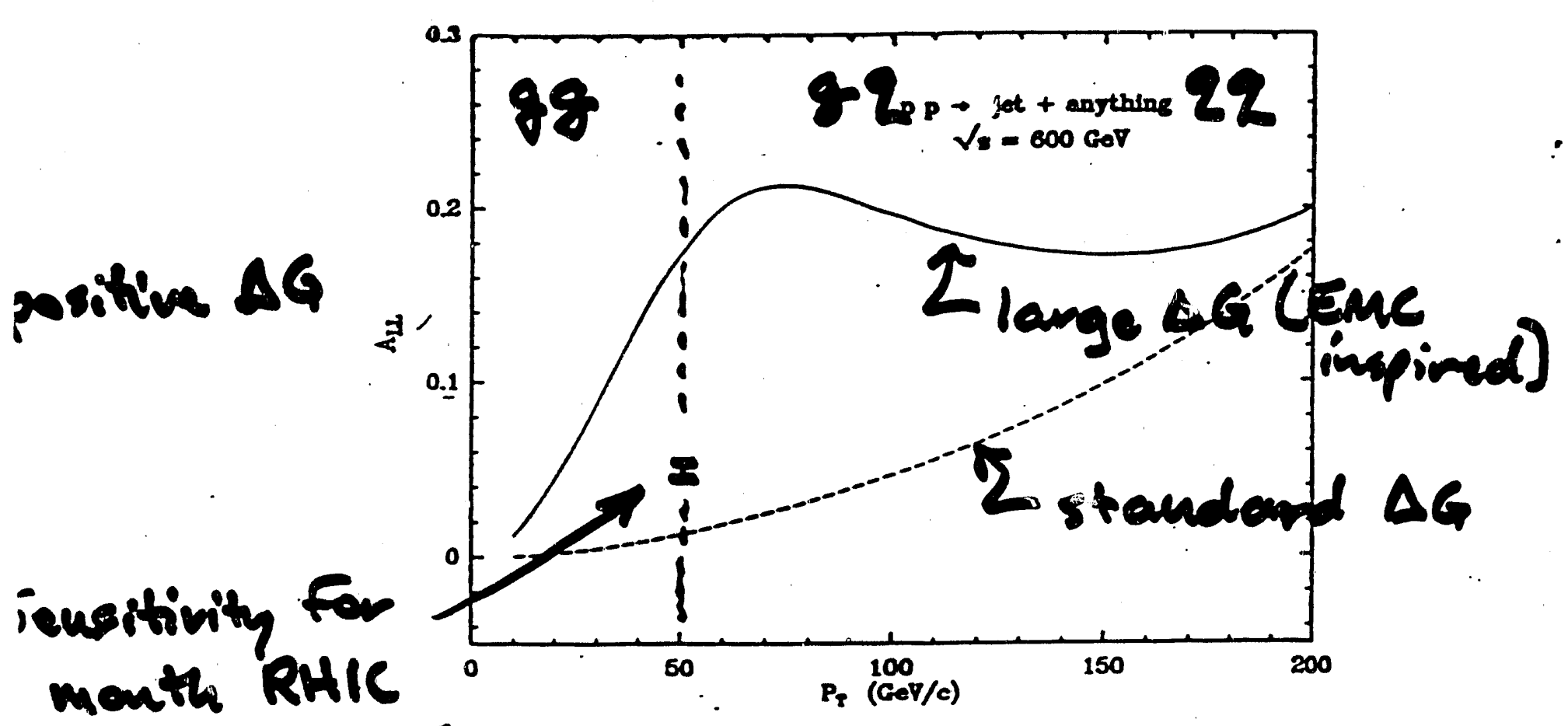

run: $\triangle A_{L 6}=.005$ a H. $^{2}=50$.

neg. $\Delta G$

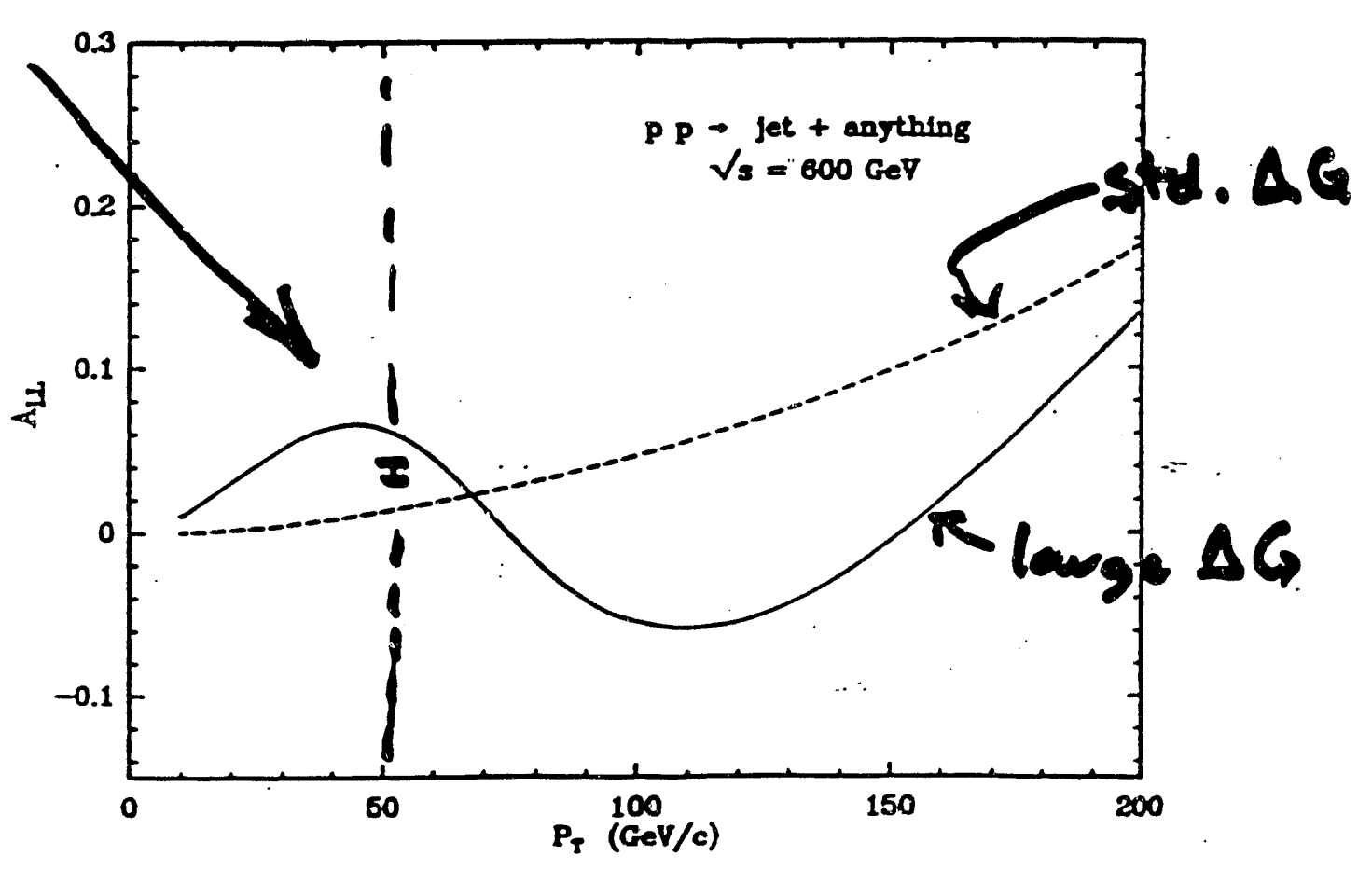

Not: $L=2 \times 10^{32}, P 1.8 .7, \Delta \ell_{p}=1$ bin, $\Delta \phi=2 \pi, y=0, \Delta y=1$. 

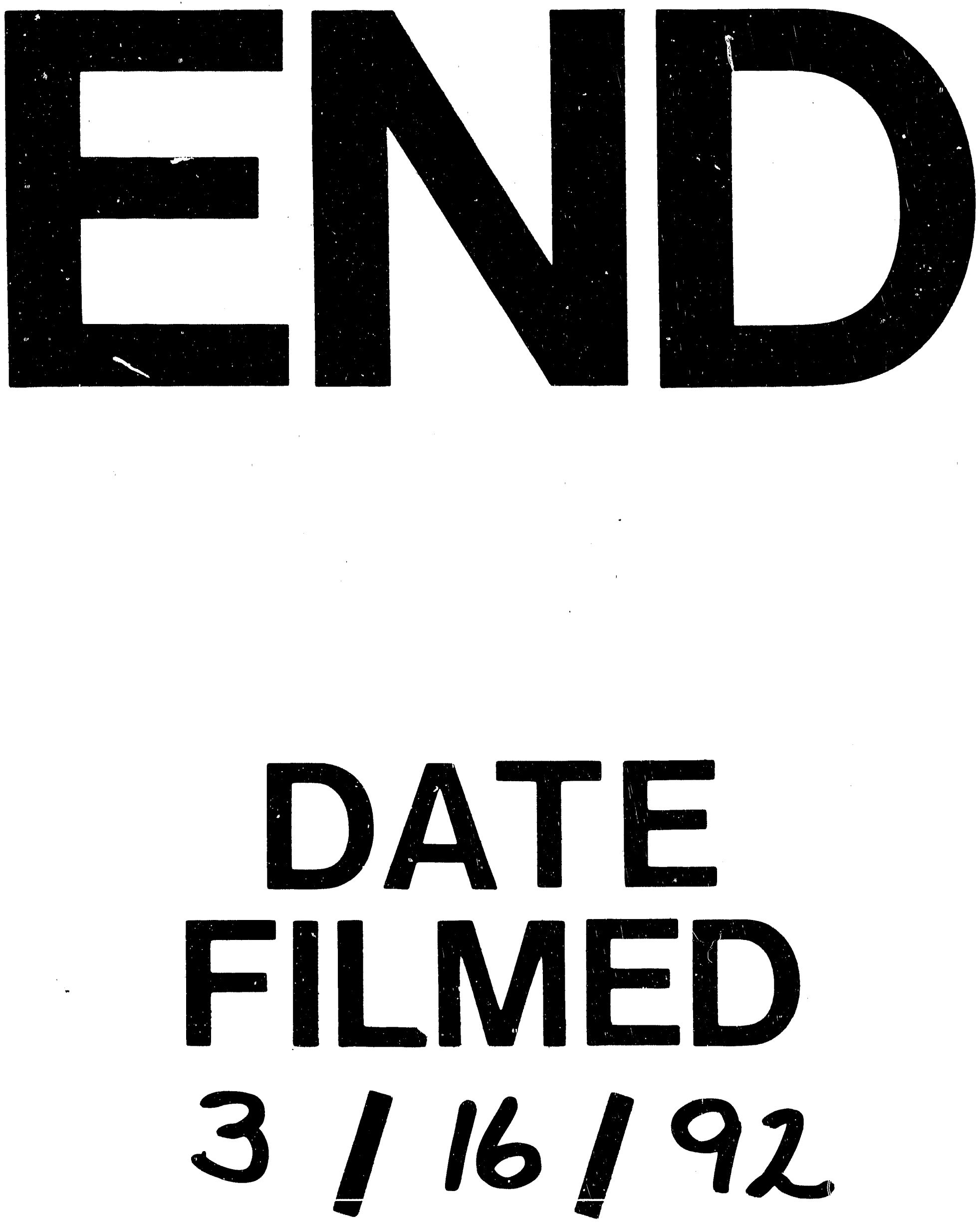

1 


$$
\text { —— }
$$

